\title{
Correlation of UGT2B7 Polymorphism with Cardiotoxicity in Breast Cancer Patients Undergoing Epirubicin/Cyclophosphamide-Docetaxel Adjuvant Chemotherapy
}

\author{
Hai Li*, Bo Hu*, Zhe Guo, Xueqing Jiang, Xinyu Su, and Xiaoyi Zhang \\ Department of Thyroid and Breast Surgery, The Central Hospital of Wuhan, Tongji Medical College, Huazhong University of Science and \\ Technology, Wuhan, China.
}

\begin{abstract}
Purpose: The present study aimed to investigate correlations between uridine glucuronosyltransferase 2B7 (UGT2B7) -161 single nucleotide polymorphism $\mathrm{C}$ to $\mathrm{T}(\mathrm{C}>\mathrm{T})$ and the occurrence of cardiotoxicity in Chinese breast cancer $(\mathrm{BC})$ patients undergoing epirubicin/cyclophosphamide-docetaxel (EC-D) adjuvant chemotherapy.

Materials and Methods: 427 BC patients who had underwent surgery were consecutively enrolled in this prospective cohort study. All patients were scheduled to receive EC-D adjuvant chemotherapy regimen, and they were divided into UGT2B7 -161 CC ( $\mathrm{n}=141)$, UGT2B7 -161 CT ( $\mathrm{n}=196)$, and UGT2B7 -161 TT ( $\mathrm{n}=90)$ groups according to their genotypes. Polymerase chain reaction was performed for determination of UGT2B7 -161 genotypes. Cardiotoxicity was defined as an absolute decline in left ventricular ejection fraction (LVEF) of at least $10 \%$ points from baseline to a value less than $53 \%$, heart failure, acute coronary artery syndrome, or fatal arrhythmia.

Results: LVEF values were lower at cycle (C) 4, C8, 3 months after chemotherapy (M3), M6, M9, and M12 compared to C0 (all $p<0.001$ ), in BC patients undergoing EC-D adjuvant chemotherapy. Cardiotoxicity was recorded for $4.2 \%$ of the overall population and was lowest in the UGT2B7 -161 TT group (1.1\%), compared to UGT2B7 -161 CT (3.1\%) and UGT2B7 -161 CC (7.8\%) group $(p=0.026)$. Multivariate logistic regression revealed that UGT2B7 -161 T allele could independently predict a low occurrence of cardiotoxicity in BC patients undergoing EC-D adjuvant chemotherapy $(p=0.004)$.

Conclusion: A UGT2B7 -161 T allele serves as a potential biomarker for predicting a low occurrence of cardiotoxicity in BC patients undergoing EC-D adjuvant chemotherapy.
\end{abstract}

Key Words: UGT2B7, polymorphism, chemotherapy, cardiotoxicity, breast cancer

Received: July 9, 2018 Revised: November 5, 2018

Accepted: November 8, 2018

Corresponding author: Xiaoyi Zhang, MM, Department of Thyroid and Breast Surgery, The Central Hospital of Wuhan, Tongji Medical College, Huazhong University of Science and Technology, 26 Shengli Street, Wuhan 430014, China. Tel: 86-27-82211427, Fax: 86-27-82211427, E-mail: zhangxiaoyee@163.com

${ }^{*}$ Hai Li and Bo Hu contributed equally to this work.

-The authors have no potential conflicts of interest to disclose.

(C) Copyright: Yonsei University College of Medicine 2019

This is an Open Access article distributed under the terms of the Creative Commons Attribution Non-Commercial License (https://creativecommons.org/licenses/ by-nc/4.0) which permits unrestricted non-commercial use, distribution, and reproduction in any medium, provided the original work is properly cited.

\section{INTRODUCTION}

Breast cancer (BC) is the most commonly diagnosed cancer in females, accounting for $25 \%$ of all cancer cases and $15 \%$ of all cancer deaths in women worldwide. Its incidence and mortality in Asia have been increasing in recent decades. ${ }^{1}$ Currently, adjuvant chemotherapy for $\mathrm{BC}$ is considered to reduce tumor recurrence and to prolong patient survival, with the development of new drugs and various chemotherapy regimens. ${ }^{2}$ Adjuvant chemotherapy using epirubicin and cyclophosphamide, followed by docetaxel (adjuvant EC-D), is the most recommended adjuvant chemotherapy regimen for eradicating residual 
tumors and eliminating post-surgical hematogenous metastasis; however it has been frequently reported to be associated with an increased risk of cardiotoxicity in BC patients. ${ }^{3-6}$ Therefore, it is necessary to explore novel biomarkers that predict the occurrence of cardiotoxicity in BC patients undergoing EC-D adjuvant chemotherapy to reduce cardiovascular morbidity and to improve quality of life.

Uridine glucuronosyltransferase 2B7 (UGT2B7), a polymorphic protein-coding gene, mediates glucuronidation to eliminate metabolites of antineoplastic drugs. ${ }^{7}$ Accumulating evidence has demonstrated that UGT2B7 gene polymorphisms regulate drugs metabolism and toxicity, thereby affecting treatment outcomes. UGT2B7 -161 (rs7668258; Genebank Ref mRNA NM_001074) single nucleotide polymorphism (SNP) C to T $(\mathrm{C}>\mathrm{T})$ has been shown to be correlated with epirubicin metabolism, toxicity, and efficacy when treating early-stage BC patients. ${ }^{8}$ However, studies of UGT2B7 -161 C $>\mathrm{T}$ in relation to cardiotoxicity in $\mathrm{BC}$ patients are lacking, particularly in Chinese patients undergoing EC-D adjuvant chemotherapy. Therefore, this prospective cohort study aimed to explore the associations of UGT2B7 polymorphism with the occurrence of cardiotoxicity in Chinese BC patients undergoing EC-D adjuvant chemotherapy.

\section{MATERIALS AND METHODS}

\section{Participants}

Between January 2013 and December 2015, 427 BC patients who had undergone surgery at The Central Hospital of Wuhan were consecutively recruited in this prospective cohort study. The inclusion criteria consisted of 1) diagnosis with primary BC with confirmed TNM stage I-III according to clinical, imaging and pathological findings; 2) age above 18 years; 3) Eastern Cooperative Oncology Group (ECOG) performance status less than or equal to $2.0 ; 4$ ) having undergone surgical treatment for $\mathrm{BC}$; 5) normal left ventricular ejection fraction (LVEF) examined by echocardiogram, defined as LVEF $\geq 55 \%$; 6 ) absence of chemotherapy contraindications; 7 ) scheduled to receive EC-D adjuvant chemotherapy; and 8) able to be followed up regularly. The exclusion criteria were as follows: 1) histologically confirmed metastatic disease (Stage IV) or bilateral BC; 2) received neoadjuvant therapy before surgery; 3 ) any previous systemic therapy (including chemotherapy, immunotherapy, human epidermal growth factor receptor-2 (HER2) targeted agents, and antitumor vaccines) or radiation therapy for other disease; 4 ) inadequate bone marrow function (absolute neutrophil count less than $1.2 \times 10^{9} / \mathrm{L}$, platelet count less than $100 \times 10^{9} / \mathrm{L}$ or haemoglobin below $10 \mathrm{~g} / \mathrm{dL}$ ) and severe renal or liver dysfunction; 5) history or evidence of cardiovascular conditions, such as congestive heart failure, cardiomyopathy, arrhythmia, myocardial infarction, etc.; 6) severe or uncontrolled systemic disease; 7) poorly controlled diabetes or evidence of clinically significant diabetic vascular complications; 8) history of severe infection, other solid tumors, or hematologic malignancy; and 9) pregnant or breast-feeding women.

\section{Ethics}

This study protocol was approved by the Ethics Committees of The Central Hospital of Wuhan (approval number ChiCTRCOC-17008261) and was conducted in accordance with the Declaration of Helsinki. All participants provided written informed consent.

\section{Date collection}

Baseline characteristics were collected from all patients after enrollment, which included age, body mass index (BMI), smoking status, hypertension, diabetes mellitus, dyslipidemia, hyperuricemia, chromic kidney disease, ECOG performance, TNM stage and levels of cardiac troponin I (cTnI) (reference range: $0-0.1 \mathrm{ng} / \mathrm{mL}$ ) and $\mathrm{N}$-terminal pro-brain natriuretic peptide (NT-proBNP) (reference range: $0-0.1 \mathrm{ng} / \mathrm{mL}$ ). Both cTnI and NT-proBNP were measured by a QMT8000 immunoassay analyzer (Wuhan Easy Diagnosis Biomedicine Co., Ltd, Wuhan, China) using rapid test kits.

\section{Determination of UGT2B7 - 161 genotype}

QIAamp DNA Mini Kits (Qiagen, Hilden, Germany) were used to extract genomic DNA from the leukocyte portion of whole blood collected from all patients prior to adjuvant chemotherapy. Then, polymerase chain reaction (PCR) was performed for the amplification of UGT2B7 -161 (rs7668258; Genebank Ref mRNA NM_001074). The PCR reactions were carried out on a Gene Amp PCR System 9700 (Applied Biosystems, Foster City, CA, USA) with an initial denaturation step at $95^{\circ} \mathrm{C}$ for $5 \mathrm{~min}$, followed by 50 cycles of $95^{\circ} \mathrm{C}$ for $15 \mathrm{sec}, 60^{\circ} \mathrm{C}$ for $30 \mathrm{sec}, 72^{\circ} \mathrm{C}$ for $30 \mathrm{sec}$, a final extension step at $72^{\circ} \mathrm{C}$ for $5 \mathrm{~min}$, and incubation at $4^{\circ} \mathrm{C}$. After that, UGT2B7 -161 was genotyped by pyrosequencing on the $\mathrm{PSQ}^{\mathrm{TM}} 96 \mathrm{MA}$ System (Qiagen) according to the manufacturer's instructions.

\section{Treatment}

After surgery, patients underwent laboratory testing and physical examination to confirm indications for chemotherapy before initiating adjuvant chemotherapy. Then, according to disease condition and patient willingness, patients received an EC-D adjuvant chemotherapy regimen, which was administrated as follows: epirubicin (E) at a dose of $100 \mathrm{mg} / \mathrm{m}^{2}$ via intravenous (IV) infusion on day 1 and cyclophosphamide (C) at a dose of $600 \mathrm{mg} / \mathrm{m}^{2}$ via IV infusion on day 1 . Both $\mathrm{E}$ and C were repeated every 21 days for four cycles, followed by docetaxel (D) $75-100 \mathrm{mg} / \mathrm{m}^{2}$ via IV infusion on day 1 every 21 days for four cycles. Patients who were HER2 positive were administrated trastuzumab at a dose of $4 \mathrm{mg} / \mathrm{kg}$ in the first week concurrently with docetaxel, followed by a dose of $2 \mathrm{mg} / \mathrm{kg}$ weekly for the next 11 weeks and a dose of $6 \mathrm{mg} / \mathrm{kg}$ every 21 days thereafter 
until completing 1 year of trastuzumab therapy.

\section{Evaluation of cardiotoxicity}

Cardiotoxicity was defined as an absolute decline in LVEF of at least 10 percentage points from baseline to a value less than $53 \%$ on an echocardiogram, ${ }^{9}$ heart failure, acute coronary artery syndrome, or fatal arrhythmia. ${ }^{10}$ LVEF was evaluated before adjuvant chemotherapy (Cycle $0, \mathrm{C} 0$ ), at the completion of four cycles of EC chemotherapy (C4), at the end of four cycles of docetaxel treatment (C8), and at 3, 6, 9, and 12 months (M3, M6, M9, and M12) after adjuvant chemotherapy, according to the American Society of Echocardiography recommendations. ${ }^{11}$

\section{Statistics analysis}

Statistical analysis was carried out with the use of SPSS 22.0 software (IBM Corp., Armonk, NY, USA) and GraphPad Prism 6.01 software (GraphPad Software Inc., La Jolla, CA, USA). Count data are expressed as percentages or rates and were compared using the chi-square test among groups. Measurement data are presented as a mean \pm standard deviation or median (interquartile range). For normal distributed data, comparison among three groups was determined by one-way ANOVA. As for the skewed distributed continuous data, comparison among three groups was determined by Kruskal-Wallis H rank sum test, and comparison at paired time point was performed using Wilcoxon signed-rank sum test. To evaluate the effect of UGT2B7 -161 T allele on cardiotoxicity, an addictive model was employed in the logistic regression model analysis of factors affecting cardiotoxicity occurrence. $p$ values $<0.05$ were considered statistically significant.

\section{RESULTS}

\section{Study flow}

In this study, $640 \mathrm{BC}$ patients who underwent surgery were initially invited to participate; 46 were excluded (31 patients missed the invitation date and 15 patients refused to attend the pre-screening procedure). Then, $594 \mathrm{BC}$ patients were screened for eligibility, and of these, 167 patients were excluded (144 patients did not meet the inclusion criteria and 23 patients were not willing to provide informed consent). Eventually, $427 \mathrm{BC}$ patients were included in the analysis with their UGT2B7 -161 genotypes determined before adjuvant chemotherapy, and the patients were assigned to CC $(\mathrm{n}=141), \mathrm{CT}(\mathrm{n}=196)$, and TT $(\mathrm{n}=90)$ groups according to their UGT2B7 -161 genotypes (Fig. 1).

\section{Correlation of UGT2B7 -161 C>T genotypes with clinical characteristics}

The mean age of the $427 \mathrm{BC}$ patients was $45.3 \pm 6.0$ years, and the median values of LVEF, cTnI, and NT-proBNP were $67.0 \%$ (64.0-71.0), 0.022 (0.010-0.056) ng/mL, and 0.076 (0.059-0.102) $\mathrm{ng} / \mathrm{mL}$, respectively. There were 141 (33.0\%), 196 (45.9\%), and

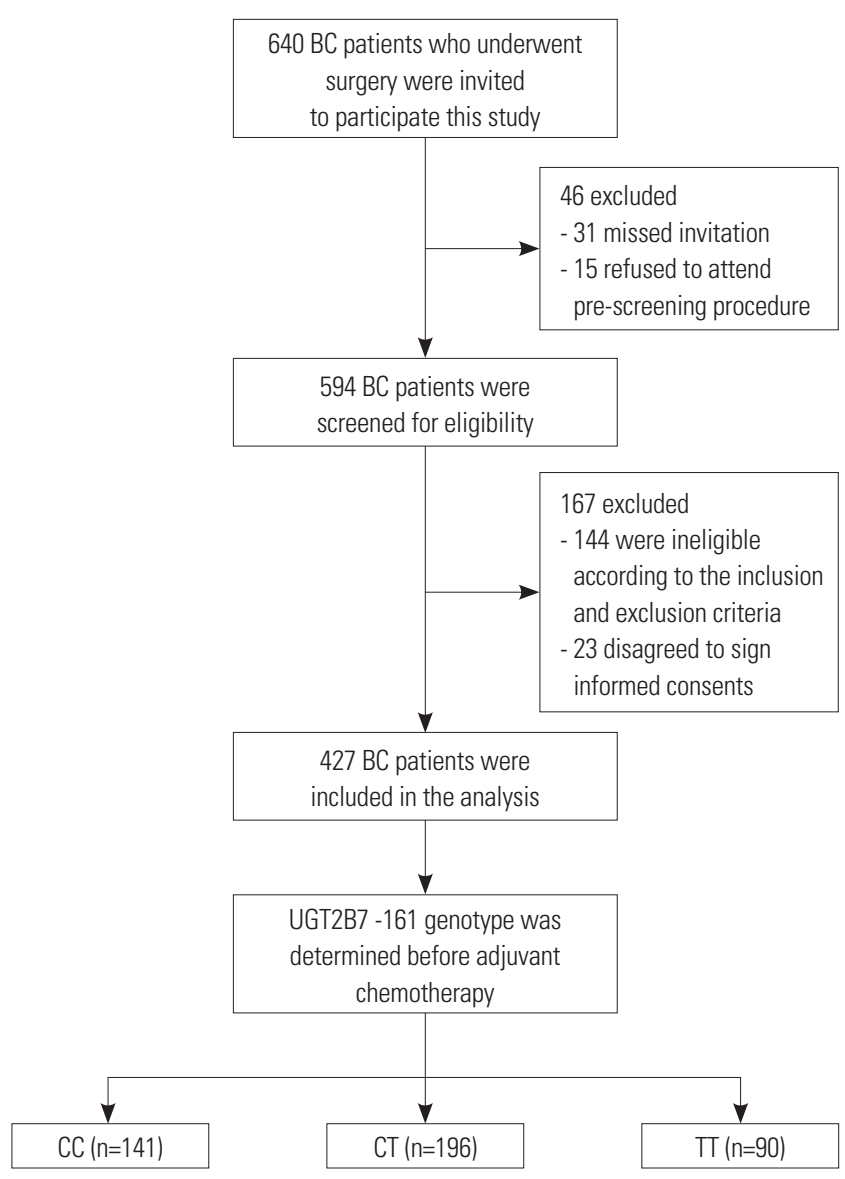

Fig. 1. Study flow. BC, breast cancer; UGT2B7, uridine glucuronosyltransferase $2 \mathrm{~B} 7$.

90 (21.1\%) patients who presented with UGT2B7 -161 CC, CT, and TT genotypes, respectively. No differences were observed in age, BMI, overweight, smoke, hypertension, diabetes mellitus, dyslipidemia, hyperuricemia, chromic kidney disease, molecular subtype, ECOG performance, TNM stage, cardiac function, cumulative dose of epirubicin, or administration of trastuzumab among BC patients with UGT2B7 -161 CC, CT, and TT genotypes (all $p>0.05$ ) (Table 1).

\section{LVEF during and after adjuvant chemotherapy in BC patients}

Median values of LVEF in BC patients at C0, C4, C8 (M0), M3, M6, M9, and M12 were 67.0\% (64.0-71.0), 64.0\% (60.0-69.0), $63.0 \%$ (58.0-67.0), 63.0\% (58.0-68.0), 65.0\% (60.0-69.0), 64.0\% (60.0-70.0), and 64.0\% (59.0-71.0), respectively (Fig. 2). Compared to $\mathrm{C} 0$, median values of $\mathrm{LVEF}$ were lower at $\mathrm{C} 4, \mathrm{C} 8(\mathrm{M} 0)$, M3, M6, M9, and M12 in BC patients undergoing EC-D adjuvant chemotherapy (all $p<0.001$ ).

\section{Cardiotoxicity after adjuvant chemotherapy in BC patients}

The percentages of cases presenting with heart failure, acute coronary syndrome, life-threatening arrhythmias, and decrease 
Hai Li, et al.

Table 1. Correlation of UGT2B7 -161 Genotype with Clinical Characteristics of Patients

\begin{tabular}{|c|c|c|c|c|c|}
\hline \multirow{2}{*}{ Clinical characteristics } & \multicolumn{5}{|c|}{ UGT2B7 rs7668258 genotype } \\
\hline & Total patients (n=427) & CC (n=141) & CT $(n=196)$ & $\Pi(n=90)$ & $p$ value \\
\hline Age (yr) & $45.3 \pm 6.0$ & $45.6 \pm 6.4$ & $45.0 \pm 6.1$ & $45.4 \pm 5.2$ & 0.669 \\
\hline $\mathrm{BMI}\left(\mathrm{kg} / \mathrm{m}^{2}\right)$ & $23.3 \pm 2.1$ & $23.3 \pm 2.1$ & $23.4 \pm 2.0$ & $23.0 \pm 2.1$ & 0.370 \\
\hline Overweight (BMl >25 kg/m²) & $82(19.2)$ & $23(16.3)$ & $46(23.5)$ & $13(14.4)$ & 0.112 \\
\hline Smoke & $104(24.4)$ & $35(24.8)$ & $43(21.9)$ & $26(28.9)$ & 0.440 \\
\hline Hypertension & $91(21.3)$ & $32(22.7)$ & $38(19.4)$ & $21(23.3)$ & 0.666 \\
\hline Diabetes mellitus & $19(4.4)$ & $6(4.3)$ & $10(5.1)$ & $3(3.3)$ & 0.790 \\
\hline Dyslipidemia & $80(18.7)$ & $23(16.3)$ & $41(20.9)$ & 16 (17.8) & 0.546 \\
\hline Hyperuricemia & $83(19.4)$ & $27(19.1)$ & 42 (21.4) & $14(15.6)$ & 0.504 \\
\hline Chromic kidney disease & $15(3.5)$ & $3(2.1)$ & $7(3.6)$ & $5(5.6)$ & 0.385 \\
\hline Molecular subtype & & & & & 0.374 \\
\hline Luminal A & $30(7.0)$ & $9(6.4)$ & $14(7.1)$ & $7(7.8)$ & \\
\hline Luminal B & 82 (19.2) & $35(24.8)$ & 31 (15.8) & $16(17.8)$ & \\
\hline ERBB2 $^{+}$ & $113(26.5)$ & $32(22.7)$ & $60(30.7)$ & $21(23.3)$ & \\
\hline Basal-like & $202(47.3)$ & $65(46.1)$ & $91(46.4)$ & 46 (51.1) & \\
\hline ECOG performance & & & & & 0.346 \\
\hline 0 & $338(79.1)$ & $106(75.2)$ & $158(80.6)$ & 74 (82.2) & \\
\hline 1 & $84(19.7)$ & $34(24.1)$ & $34(17.4)$ & $16(17.8)$ & \\
\hline 2 & $5(1.2)$ & $1(0.7)$ & $4(2.0)$ & $0(0.0)$ & \\
\hline TNM stage & & & & & 0.786 \\
\hline I & $49(11.5)$ & $16(11.3)$ & $20(10.2)$ & $13(14.4)$ & \\
\hline$\|$ & $268(62.8)$ & $91(64.5)$ & $125(63.8)$ & $52(57.8)$ & \\
\hline III & $110(25.8)$ & $34(24.1)$ & $51(26.0)$ & $25(27.8)$ & \\
\hline \multicolumn{6}{|l|}{ Cardiac function } \\
\hline $\operatorname{LVEF}(\%)$ & $67.0(64.0-71.0)$ & $67.0(63.5-70.0)$ & $67.0(64.0-71.0)$ & $67.5(64.0-70.3)$ & 0.747 \\
\hline$c T n l(n g / m L)$ & $0.022(0.010-0.056)$ & $0.022(0.010-0.058)$ & $0.023(0.011-0.059)$ & 0.017 (0.008-0.045) & 0.137 \\
\hline NT-proBNP (ng/mL) & $0.076(0.059-0.102)$ & 0.079 (0.059-0.119) & $0.073(0.057-0.095)$ & $0.077(0.061-0.101)$ & 0.076 \\
\hline Cumulative dose of epirubicin $\left(\mathrm{mg} / \mathrm{m}^{2}\right)$ & $302.0(281.0-321.0)$ & $301.0(282.5-320.0)$ & $301.0(276.0-322.5)$ & $304.0(286.0-323.2)$ & 0.425 \\
\hline Administration of trastuzumab & $102(23.9)$ & $32(22.7)$ & $44(22.4)$ & $26(28.9)$ & 0.456 \\
\hline
\end{tabular}

BMI, body mass index; ECOG, Eastern Cooperative Oncology Group; LVEF, left ventricular ejection fraction; cTnl, cardiac troponin I; NT-proBNP, N-terminal probrain natriuretic peptide; UGT2B7, uridine glucuronosyltransferase 2B7.

Data are presented as a mean \pm standard deviation, median (interquartile range), or count (\%). Comparison was determined by one-way ANOVA, Kruskal-Wallis

$\mathrm{H}$ rank sum test, or chi-square test. $p$ value $<0.05$ was considered significant.

in $\mathrm{LVEF} \geq 10 \%$ to absolute $<53 \%$ were $0.5,0.0,0.2$, and $4.0 \%$, respectively (two patients presented with both heart failure and LVEF decreased $\geq 10 \%$ to absolute $<53 \%$ ). The total occurrence of cardiotoxicity was $4.2 \%$ (Fig. $3 \mathrm{~A}$ ). The occurrence rate of cardiotoxicity was reduced as more patients carried UGT2B7 $-161(p=0.026)$ (Fig. 3B).

\section{Factors affecting cardiotoxicity occurrence by univariate logistic regression model analysis in the addictive model}

Univariate logistic regression revealed that UGT2B7 -161 T allele was correlated with a low occurrence of cardiotoxicity ( $p=$ 0.013), whereas high cTnI concentration $(>0.022 \mathrm{ng} / \mathrm{mL})(p=$ $0.003)$, high NT-proBNP concentration $(>0.076 \mathrm{ng} / \mathrm{mL})(p=$ 0.023 ), and adminstration of trastuzumab ( $p=0.044)$ were correlated with increased occurrence of cardiotoxicity in BC patients undergoing EC-D chemotherapy after surgery (Table 2).
Factors affecting cardiotoxicity occurrence by multivariate logistic regression model analysis in the addictive model

Multivariate logistic regression indicated that a UGT2B7 -161 $\mathrm{T}$ allele could independently predict a low occurrence of cardiotoxicity in BC patients undergoing EC-D chemotherapy $(p=0.004)$. However, high cTnI concentration $(>0.022 \mathrm{ng} / \mathrm{mL})$ ( $p=0.002)$, high NT-proBNP concentration $(>0.076 \mathrm{ng} / \mathrm{mL})$ $(p=0.035)$, high cumulative dose of epirubicin $\left(>302 \mathrm{mg} / \mathrm{m}^{2}\right)$ $(p=0.048)$, and administration of trastuzumab $(p=0.032)$ were independent risk factors for cardiotoxicity in BC patients undergoing EC-D chemotherapy (Table 3).

\section{DISCUSSION}

In this prospective study, we observed that 1) LVEF is reduced 
in BC patients during and after EC-D adjuvant chemotherapy and that the occurrence rate of cardiotoxicity is $4.2 \%$. 2) A UGT2B7 -161 T allele independently predicts a low occurrence of cardiotoxicity in Chinese BC patients undergoing EC-D adjuvant chemotherapy.

Cardiotoxicity is one of the most common adverse effects in BC patients receiving anthracycline-based adjuvant therapies, which has been widely investigated in various studies. For example, a retrospective cohort study demonstrated that risk of heart failure is increased in BC patients receiving anthracycline-based adjuvant chemotherapy, compared to non-che-

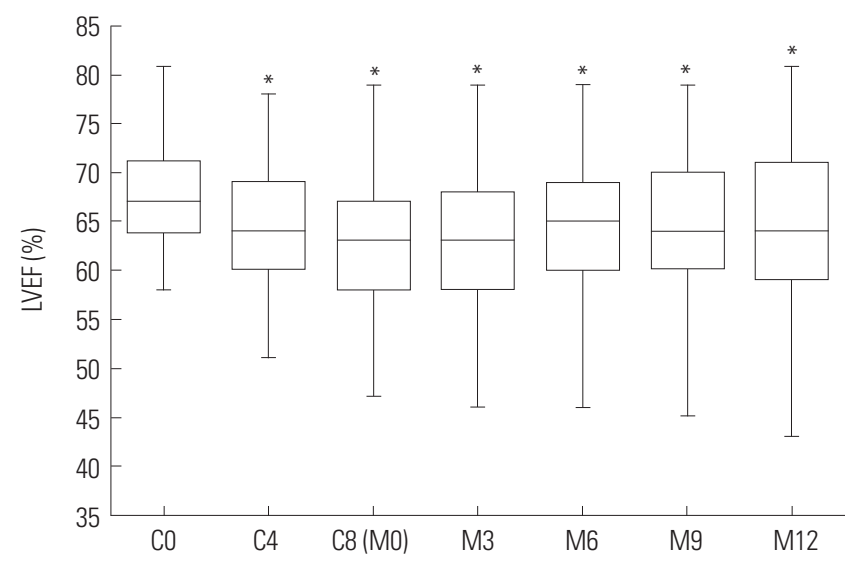

Fig. 2. LVEF during and after adjuvant chemotherapy in BC patients. LVEF values were lower at $\mathrm{C} 4, \mathrm{C} 8$ (M0), $\mathrm{M} 3, \mathrm{M} 6, \mathrm{M} 9$, and $\mathrm{M} 12$, compared to $\mathrm{CO}$ in $\mathrm{BC}$ patients undergoing $\mathrm{EC}-\mathrm{D}$ adjuvant chemotherapy. Comparison between paired time points was performed using Wilcoxon signed-rank sum test. ${ }^{*} p<0.001$ compared with CO. LVEF, left ventricular ejection fraction; C4/C8, 4th/8th cycle of adjuvant chemotherapy; M3/M6/M9/M12, $3 / 6 / 9 / 12$ months after adjuvant chemotherapy; $\mathrm{BC}$, breast cancer.

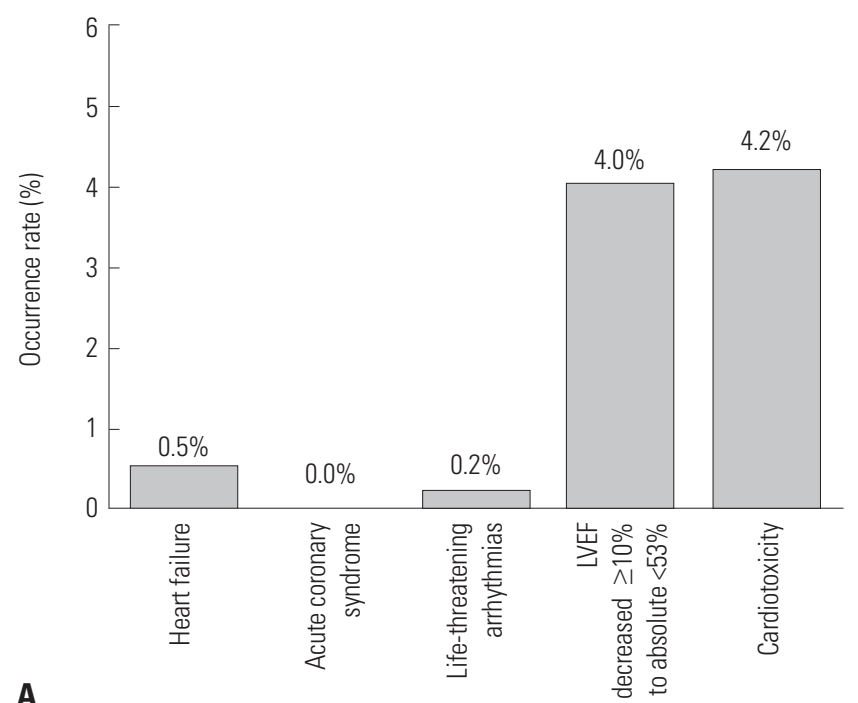

motherapy group..$^{12}$ Also, a low dose of epirubicin in adjuvant chemotherapy was found to result in less decline in LVEF and to lower the risk of cardiac damage in BC patients..$^{13}$ Another study revealed that the risk of cardiotoxicity is higher in HER2possitive $\mathrm{BC}$ patients undergoing doxorubicin and cyclophosphamide, followed by docetaxel plus trastuzumab, compared with that in patients receiving non-anthracycline-combined trastuzumab adjuvant therapy. ${ }^{14}$ Accordingly, this evidence suggests that anthracyclines-based adjuvant chemotherapy may cause an increased risk of cardiotoxicity in BC patients. In line with these previous studies, our study observed that LVEF was decreased during and after adjuvant chemotherapy, and the occurrence of cardiotoxicity was $4.2 \%$ in $427 \mathrm{BC}$ patients receiving EC-D adjuvant chemotherapy. This might be because drugs or metabolites of anthracyclines, such as epirubicin, in an EC-D regimen are toxic to cardiac cells, which lead to impairment of cardiac cell function, thereby decreasing LVEF and increasing the risk of cardiotoxicity in BC patients receiving EC-D adjuvant chemotherapy. Epirubicin is a derivative of doxorubicin, which is the most commonly reported anthracycline leading to cardiotoxicity, and studies comparing epirubicin and doxorubicin-induced cardiotoxicity in BC patients have revealed a difference when doses are high. ${ }^{15,16}$ Nevertheless, our data on the cardiotoxicity of epirubicin cannot be extrapolated to doxorubicin, although these two drugs have similar mechanisms in the treatment of BC.

UGT2B7 is a hepatic enzyme that plays an important role in mediating glucuronidation to eliminate antineoplastic drugs, as well as their active metabolites, and its polymorphism has been reported to alter glucuronidation ability and to affect metabolism and toxicity of drugs used for BC adjuvant therapy. ${ }^{17}$

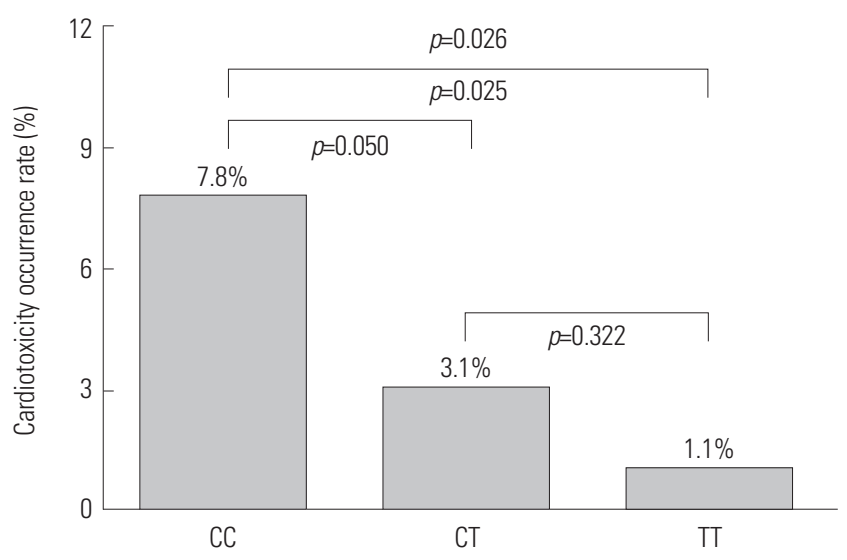

B

Fig. 3. Occurrence of cardiotoxicity after adjuvant chemotherapy in BC patients. (A) The total occurrence of cardiotoxicity was $4.2 \%$ in $427 \mathrm{BC}$ patients, and the percentage of cases presenting with heart failure, acute coronary syndrome, life-threatening arrhythmias, and LVEF decreased $\geq 10 \%$ to absolute $<53 \%$ were $0.5,0.0,0.2$, and $4.0 \%$, respectively (Two patients presented with both heart failure and LVEF decreased $\geq 10 \%$ to absolute $<53 \%$ ). (B) The occurrence rate of cardiotoxicity in patients with UGT2B7 -161 TT genotype was 1.1\%, which was lower than that in patients with UGT2B7 -161 CT (3.1\%) and UGT2B7 -161 CC (7.8\%) genotypes. Comparison among three groups was conducted using chi-square test, and $p<0.05$ was considered significant. $\mathrm{BC}$, breast cancer; LVEF, left ventricular ejection fraction; UGT2B7, uridine glucuronosyltransferase $2 \mathrm{~B} 7$. 
Hai Li, et al.

An interesting study on Thai BC patients with impaired CYP3D6 discovered that UGT2B7 $802 \mathrm{C}>\mathrm{T}$ polymorphism reduces the metabolism of tamoxifen as adjuvant hormonal therapy, as well as the active metabolite endoxifen, by decreasing gluc- uronidation. ${ }^{18}$ As to the association of UGT2B7 polymorphism with epirubicin metabolism, a recent study disclosed that UGT2B7 802 C > T leads to lowered UGT glucuronidation capacity and delays the elimination of adjuvant epirubicin, as

Table 2. Factors Affecting Cardiotoxicity Occurrence in Univariate Logistic Regression Model Analysis (Addictive Model)

\begin{tabular}{|c|c|c|c|c|}
\hline \multirow{3}{*}{ Parameters } & \multicolumn{4}{|c|}{ Univariate logistic regression } \\
\hline & \multirow{2}{*}{$p$ value } & \multirow{2}{*}{ OR } & \multicolumn{2}{|c|}{$95 \% \mathrm{Cl}$} \\
\hline & & & Lower & Higher \\
\hline UGT2B7 -161 T allele (addictive model: CC-0, CT-1, TT-2) & 0.013 & 0.369 & 0.168 & 0.808 \\
\hline Age $(>45 \mathrm{yr})$ & 0.541 & 1.345 & 0.520 & 3.477 \\
\hline Overweight (BMl >25 kg/m²) & 0.350 & 1.658 & 0.574 & 4.790 \\
\hline Smoke & 0.368 & 1.587 & 0.580 & 4.339 \\
\hline Hypertension & 0.923 & 1.057 & 0.340 & 3.294 \\
\hline Diabetes mellitus & 0.817 & 1.278 & 0.161 & 10.140 \\
\hline Dyslipidemia & 0.320 & 1.713 & 0.593 & 4.951 \\
\hline Hyperuricemia & 0.365 & 1.632 & 0.565 & 4.713 \\
\hline Chromic kidney disease & 0.634 & 1.660 & 0.206 & 13.365 \\
\hline Higher ECOG performance & 0.984 & 1.011 & 0.349 & 2.932 \\
\hline Higher TNM stage & 0.325 & 1.498 & 0.669 & 3.355 \\
\hline LVEF high (>67.0\%) & 0.203 & 0.523 & 0.192 & 1.419 \\
\hline cTnl high (>0.022 ng/mL) & 0.003 & 9.221 & 2.093 & 40.620 \\
\hline NT-proBNP high (>0.076 ng/mL) & 0.023 & 3.693 & 1.196 & 11.411 \\
\hline Cumulative dose of epirubicin high (>302 mg/m²) & 0.110 & 2.260 & 0.832 & 6.139 \\
\hline Administration of trastuzumab & 0.044 & 2.681 & 1.029 & 6.986 \\
\hline
\end{tabular}

UGT2B7, uridine glucuronosyltransferase 2B7; BMI, body mass index; ECOG, Eastern Cooperative Oncology Group; LVEF, left ventricular ejection fraction; cTnl, cardiac troponin I; NT-proBNP, N-terminal pro-brain natriuretic peptide; $\mathrm{OR}$, odds ratio; $\mathrm{Cl}$, confidence interval.

Data are presented as a $p$ value or OR and 95\% CI. UGT2B7 -161 genotype was analyzed using an addictive model, which was scored as CC-0, CT-1, and TT-2. All continuous variables were stratified by medians respectively. $p$ value $<0.05$ was considered significant.

Table 3. Factors Affecting Cardiotoxicity Occurrence in Multivariate Logistic Regression Model Analysis (Addictive Model)

\begin{tabular}{|c|c|c|c|c|}
\hline \multirow{3}{*}{ Parameters } & \multicolumn{4}{|c|}{ Multivariate logistic regression } \\
\hline & \multirow{2}{*}{$p$ value } & \multirow{2}{*}{ OR } & \multicolumn{2}{|c|}{$95 \% \mathrm{CI}$} \\
\hline & & & Lower & Higher \\
\hline UGT2B7 -161 T allele (addictive model: CC-0, CT-1, TT-2) & 0.004 & 0.259 & 0.103 & 0.651 \\
\hline Age (>45 yr) & 0.675 & 1.267 & 0.419 & 3.826 \\
\hline Overweight (BMl >25 kg/m²) & 0.262 & 2.130 & 0.569 & 7.970 \\
\hline Smoke & 0.085 & 3.468 & 0.843 & 14.266 \\
\hline Hypertension & 0.199 & 0.375 & 0.084 & 1.675 \\
\hline Diabetes mellitus & 0.662 & 1.755 & 0.141 & 21.781 \\
\hline Dyslipidemia & 0.639 & 1.380 & 0.359 & 5.299 \\
\hline Hyperuricemia & 0.550 & 1.533 & 0.377 & 6.233 \\
\hline Chromic kidney disease & 0.142 & 9.087 & 0.479 & 172.445 \\
\hline Higher ECOG performance & 0.703 & 0.774 & 0.207 & 2.890 \\
\hline Higher TNM stage & 0.235 & 1.779 & 0.687 & 4.608 \\
\hline LVEF high (>67.0\%) & 0.764 & 1.217 & 0.338 & 4.385 \\
\hline cTnl high (>0.022 ng/mL) & 0.002 & 16.124 & 2.691 & 96.614 \\
\hline NT-proBNP high (>0.076 ng/mL) & 0.035 & 4.156 & 1.108 & 15.592 \\
\hline Cumulative dose of epirubicin high $\left(>302 \mathrm{mg} / \mathrm{m}^{2}\right)$ & 0.048 & 3.324 & 1.009 & 10.950 \\
\hline Administration of trastuzumab & 0.032 & 3.612 & 1.113 & 11.715 \\
\hline
\end{tabular}

UGT2B7: uridine glucuronosyltransferase 2B7, BMI: body mass index; ECOG, Eastern Cooperative Oncology Group; LVEF, left ventricular ejection fraction; cTnl, cardiac troponin I; NT-proBNP, N-terminal pro-brain natriuretic peptide; OR, odds ratio; Cl, confidence interval.

Data are presented as a $p$ value or OR and 95\% CI. UGT2B7 -161 genotype was analyzed using an addictive model, which was scored as CC-0, CT-1, or TT-2. All continuous variables were stratified by medians respectively. $p$ value $<0.05$ was considered significant. 
well as its active metabolites, in BC patients. ${ }^{7}$ Also, UGT2B7 $-161 \mathrm{C}>\mathrm{T}$ has been shown to be located on promoter region, and UGT2B7 -161 TT homozygote presents with higher UGT glucuronidation ability than UGT2B7 -161 CT heterozygote or UGT2B7 -161 CC homozygote. UGT2B7 -161 TT homozygote appears to increase the clearance of epirubicin and decrease the risk of myelosuppression, compared to UGT2B7 -161 CC homozygotes, in BC patients. ${ }^{8,19}$ Therefore, these previous studies imply that UGT2B7 polymorphisms alter the glucuronidation ability of UGT enzyme to affect drug metabolism, including epirubicin. Few clinical studies, however, have investigated the correlation of UGT2B7 polymorphisms with drug adverse effects in BC patients undergoing EC-D chemotherapy. Considering that UGT2B7 -161 C>T was located on the promoter region of UGT2B7 and that UGT glucuronidation is the main inactivation pathway of epirubicin, we assumed that UGT2B7 -161 C>T may influence the occurrence of cardiotoxicity in BC patients receiving EC-D adjuvant chemotherapy. In this study, we discovered that UGT2B7 -161 TT homozygote carriers expereience lower rates of cardiotoxicity than CT heterozygote and CC homozygote carriers. Interestingly, UGT2B7 -161 T allele appeared to independently predict a low occurrence of cardiotoxicity in BC patients undergoing EC-D chemotherapy. The possible explanation may be that UGT2B7 -161 C>T is located on the promoter region and activates the transcription of UGT2B7 protein to increase UGT enzyme activity, as well as the glucuronidation of epirubicin in EC-D adjuvant chemotherapy, protecting cardiac cells from epirubicin and its toxic metabolites, thereby reducing the risk of cardiotoxicity in $\mathrm{BC}$ patients. However, the underlying mechanism of UGT emzymatic activation and glucuronidation by UGT2B7 -161 C>T needs further validation.

There were several limitations in our study. 1) All patients in this study were Chinese and from a single center: UGT2B7 -161 SNP is also seen in other Asian population, as well as European and Sub-Saharan African. ${ }^{20}$ Therefore, our study was subject to selection bias. 2) The smple size in this study was relatively small, which reduced the statistical power. 3) The follow-up peroid for cardiotoxicity in BC patients after EC-D adjuvant chemotherapy was only 12 months, and the correlation of UGT2B7 -161 C>T with long-term cardiotoxicity was not investigated.

In conclusion, we found a UGT2B7 -161 T allele to serve as a potential biomarker for predicting low occurrence of cardiotoxicity in BC patients undergoing EC-D adjuvant chemotherapy.

\section{ORCID iDs}

Hai $\mathrm{Li}$

Bo Hu

Zhe Guo

Xueqing Jiang

Xinyu Su

Xiaoyi Zhang https://orcid.org/0000-0002-3304-1429 https://orcid.org/0000-0003-3658-1188 https://orcid.org/0000-0001-8983-1975 https://orcid.org/0000-0001-5273-8929 https://orcid.org/0000-0003-4479-0078 https://orcid.org/0000-0002-4391-0616

\section{REFERENCES}

1. Torre LA, Bray F, Siegel RL, Ferlay J, Lortet-Tieulent J, Jemal A. Global cancer statistics, 2012. CA Cancer J Clin 2015;65:87-108.

2. Jiang L, Jing C, Kong X, Li X, Ma T, Huo Q, et al. Comparison of adjuvant ED and EC-D regimens in operable breast invasive ductal carcinoma. Oncol Lett 2016;12:1448-54.

3. Ejlertsen B, Tuxen MK, Jakobsen EH, Jensen MB, Knoop AS, Højris I, et al. Adjuvant cyclophosphamide and docetaxel with or without epirubicin for early TOP2A-normal breast cancer: DBCG 07-READ, an open-label, phase III, randomized trial. J Clin Oncol 2017;35:2639-46.

4. Domercant J, Polin N, Jahangir E. Cardio-oncology: a focused review of anthracycline-, human epidermal growth factor receptor 2 inhibitor-, and radiation-induced cardiotoxicity and management. Ochsner J 2016;16:250-6.

5. Ky B, Putt M, Sawaya H, French B, Januzzi JL Jr, Sebag IA, et al. Early increases in multiple biomarkers predict subsequent cardiotoxicity in patients with breast cancer treated with doxorubicin, taxanes, and trastuzumab. J Am Coll Cardiol 2014;63:809-16.

6. Appel JM, Sogaard P, Mortensen CE, Skagen K, Nielsen DL. Tissue-Doppler assessment of cardiac left ventricular function during short-term adjuvant epirubicin therapy for breast cancer. J Am Soc Echocardiogr 2011;24:200-6.

7. Parmar S, Stingl JC, Huber-Wechselberger A, Kainz A, Renner W, Langsenlehner U, et al. Impact of UGT2B7 His268Tyr polymorphism on the outcome of adjuvant epirubicin treatment in breast cancer. Breast Cancer Res 2011;13:R57.

8. Sawyer MB, Pituskin E, Damaraju S, Bies RR, Vos LJ, Prado CM, et al. A uridine glucuronosyltransferase 2B7 polymorphism predicts epirubicin clearance and outcomes in early-stage breast cancer. Clin Breast Cancer 2016;16:139-44.

9. Lang RM, Badano LP, Mor-Avi V, Afilalo J, Armstrong A, Ernande $\mathrm{L}$, et al. Recommendations for cardiac chamber quantification by echocardiography in adults: an update from the American Society of Echocardiography and the European Association of Cardiovascular Imaging. J Am Soc Echocardiogr 2015;28:1-39.

10. Kitayama H, Kondo T, Sugiyama J, Kurimoto K, Nishino Y, Kawada M, et al. High-sensitive troponin T assay can predict anthracycline- and trastuzumab-induced cardiotoxicity in breast cancer patients. Breast Cancer 2017;24:774-82.

11. Stewart GP, Bagley RL, Froemling RA. Evaluation of reversible hydrocolloid impression material in a wet field. J Prosthet Dent 1984; 51:797-800.

12. Bowles EJ, Wellman R, Feigelson HS, Onitilo AA, Freedman AN, Delate T, et al. Risk of heart failure in breast cancer patients after anthracycline and trastuzumab treatment: a retrospective cohort study. J Natl Cancer Inst 2012;104:1293-305.

13. Meinardi MT, van Veldhuisen DJ, Gietema JA, Dolsma WV, Boomsma F, van den Berg MP, et al. Prospective evaluation of early cardiac damage induced by epirubicin-containing adjuvant chemotherapy and locoregional radiotherapy in breast cancer patients. J Clin Oncol 2001;19:2746-53.

14. Slamon D, Eiermann W, Robert N, Pienkowski T, Martin M, Press M, et al. Adjuvant trastuzumab in HER2-positive breast cancer. N Engl J Med 2011;365:1273-83.

15. Jain KK, Casper ES, Geller NL, Hakes TB, Kaufman RJ, Currie V, et al. A prospective randomized comparison of epirubicin and doxorubicin in patients with advanced breast cancer. J Clin Oncol 1985;3:818-26.

16. Cottin Y, Touzery C, Dalloz F, Coudert B, Toubeau M, Riedinger A, et al. Comparison of epirubicin and doxorubicin cardiotoxicity 
induced by low doses: evolution of the diastolic and systolic parameters studied by radionuclide angiography. Clin Cardiol 1998;21:665-70.

17. Innocenti F, Iyer L, Ramírez J, Green MD, Ratain MJ. Epirubicin glucuronidation is catalyzed by human UDP-glucuronosyltransferase 2B7. Drug Metab Dispos 2001;29:686-92.

18. Areepium N, Panomvana D, Rungwanonchai P, Sathaporn S, Voravud N. Effects of CYP2D6 and UGT2B7 polymorphisms on pharmacokinetics of tamoxifen in Thai breast cancer patients.
Breast Cancer (Dove Med Press) 2013;5:73-8.

19. Bastami S, Gupta A, Zackrisson AL, Ahlner J, Osman A, Uppugunduri S. Influence of UGT2B7, OPRM1 and ABCB1 gene polymorphisms on postoperative morphine consumption. Basic Clin Pharmacol Toxicol 2014;115:423-31.

20. National Center for Biotechnology Information. Reference SNP (refSNP) cluster report: rs7668258 [accessed on 2018 April 2]. Available at: https://www.ncbi.nlm.nih.gov/projects/SNP/snp ref.cgi?rs=7668258. 